\title{
Dos conferencias sobre la hegemonía cultural de la lengua inglesa
}

\author{
JOSEPH BOYLE \\ Universidad de Hong Kong
}

En las siguientes páginas incluimos la transcripción de dos conferencias dictadas sobre el lingüista Joseph Boyle acerca de las connotaciones que tiene la preeminencia de la lengua inglesa en el contexto mundial. Las conferencias fueron dictadas en nuestra Universidad, los días 11 y 13 de marzo del corriente año. Debemos la traducción y la transcripción de las mismas a la valiosa iniciativa de Ana Maria Martínez, docente del Departamento de Idiomas, quien sirvió de intérprete para la intervención del autor.

\section{Primera conferencia: \\ El IMPERIALISMO LINGÜÍSTICO Y EL IDIOMA INGLÉS}

\section{Introducción}

Ahora les hablaré sobre la noción del imperialismo lingüístico y el idioma inglés. Aunque será necesario algún análisis teórico, aplicaré la teoría a situaciones lingüisticas de la vida real. Sin embargo no hablaré sobre la situación lingüística que se experimenta en América Latina,

Dos conferencios sobre la hegemonía cultural de la lengua ingleso 
ya que ustedes conocen mucho más sobre el asunto que yo. A pesar de que viví en América Latina durante cinco años, esto fue en Guyana, donde el idioma nativo era el inglés y no el español.

Los países que usaré como ejemplo serán lugares de los que he tenido un conocimiento y una experiencia directas. Por ello, en la conferencia de hoy, sobre el imperialismo lingüístico y el idioma inglés, tomaré el ejemplo de Hong Kong, que conozco bien, puesto que he trabajado en el Departamento de Inglés de la Universidad de China durante veintitrés años.

En mi segunda conferencia, en la que trataré sobre las variaciones más internacionales del inglés y las actitudes cambiantes hacia el inglés, tomaré como ejemplos a tres países con los que estoy muy familiarizado: China, la India y las Filipinas. China recobró la soberanía de la colonia británica de Hong Kong en 1997; la India constituyó una de las colonias más grandes de Gran Bretaña; y las Filipinas fueron una colonia de los Estados Unidos (aunque algunos podrían cuestionar el fue). Desde 1997, Hong Kong es una Región Administrativa Especial de China, y por lo tanto estamos muy bien informados de lo que está pasando en ese país. También conozco considerablemente bien la India, las Filipinas, por haber trabajado en esos países durante un año, y haber viajado muy extensamente en las regiones de los diferentes idiomas de estos países.

Retomemos pues, el tema de ahora, el imperialismo lingüístico y el idioma inglés, con referencia especial al ejemplo de Hong Kong.

\section{El inglés, la lengua franca del mundo}

Nos guste o no, y para disgusto de los franceses, cuyo idioma fue en algún momento el idioma diplomático del mundo, el inglés se ha convertido ahora en la lengua franca global. El término lengua franca generalmente es mal entendido como si tuviera algo que ver con Francia. De hecho, originalmente simbolizó una especie de italiano, mezclado con francés, griego, árabe y otros fragmentos de idiomas, que se hablaba en las costas del Mediterráneo. En la actualidad, el término ha llegado a entenderse como cualquier lenguaje o mezcla de lenguajes que sirven como un medio común de comunicación entre personas con idiomas nativos diferentes. 
Cuando el término lengua franca se usa para el inglés, no se refiere a ninguno de los ingleses rudimentarios y criollos esparcidos por el mundo, sino que a alguna forma de inglés estándar que puede ser usada como un medio adecuado de comunicación internacional.

A pesar del comprensible resentimiento de algunas naciones ante la aparente arrogancia lingüística de las naciones poderosas de habla inglesa, no obstante, el inglés ha sido reconocido como el medio de difusión lingüística común del mundo por veinte años.

Como lo enunciara la revista Newsweek en 1982: "Ahora, les guste o no, el inglés es la cosa más cercana a la lengua franca alrededor del planeta" (Treen, 1982). Diez años atrás, leíamos en The International Herald Tribune: "De hecho, el inglés se ha convertido en la lengua franca de los negocios y de la política, desde Berlin hasta Bangkok" (Estrada, 1992). Y tres años antes, en Time: ¿Por qué pelear para preservar los idiomas pequeños cuando la lengua franca de la economía global es el inglés? (Ignatieff, 1995).

Cabe hacer notar por supuesto, que estas tres citas vienen de publicaciones de los Estados Unidos, un país que tiende a tomar por sentado que en las comunicaciones internacionales son los otros los que deben hablar su idioma. Sin embargo, las publicaciones lingüísticas británicas también hacen eco del mismo tema, con The Linguist, la publicación del Instituto de Lingüística en Londres, al afirmar categóricamente: "El inglés se ha convertido en la única lengua franca en el mundo" (Newmark, 2000), y en English Today, de Cambridge University Press, al establecer con franqueza, si bien más modestamente: "el inglés, el idioma internacional, con su base impresa, es, al final del siglo $\mathrm{xx}$, un logro marcado al servicio de toda la raza humana como la primera lengua franca global de alto nivel. Si tal entidad no existiera todavía, la aldea global hubiera tenido que inventarla. (McArthur, 1997).

El idioma inglés en el mundo moderno ha asumido un estatus multinacional y como otros entes multinacionales, tiene sus defensores y sus críticos. Sus defensores ven al inglés como una fuerza positiva para el entendimiento internacional en un mundo dividido. Sus críticos lo ven como una herramienta neoimperialista de las multinacionales, destructora de los idiomas y de las culturas locales. La acusación de "imperialismo lingüístico", levantada en su contra, obedece princi- 
palmente a su asociación con el poderío empresarial y militar angloamericano y a su implacable expansión mundial.

Ciertamente, entre los lingüistas británicos se han admitido francamente las oportunidades económicas que ofrece la exportación del inglés británico: "De alguna manera, Gran Bretaña ha podido cultivar la noción de que ella es la guardiana del buen gusto. Esta es una noticia económica buena para Gran Bretaña, al instituir al idioma inglés como un mercado más importante, con un flujo de estudiantes de todas partes del mundo llegando en bandadas a este país para tomar cursos en una desconcertante variedad de escuelas y universidades" (Sinclair, 1988: 5).

Un reporte del Concilio Británico se refirió al motivo económico de forma más evidente: "Nuestro lenguaje es nuestro bien mayor, aún más que el petróleo del Mar del Norte (el suministro), es inagotable; y es más, aún cuando no tenemos un monopolio, nuestra marca en particular es considerablemente cotizada y necesitamos invertir en él y explotar al máximo este beneficio que Dios nos dio" (Informe del Concilio Británico, 1983: 4).

Esta estrategia ha resultado exitosa; un estimado reciente del valor del mercado del inglés lo atestigua: "No es accidental el que una de las industrias prósperas de Gran Bretaña sea la enseñanza del inglés: El Reino Unido gana más de $\$ 3$ billones al año enseñándole inglés a 600,000 estudiantes anualmente". (Hutton, 1999: 14) Y el mercado se está expandiendo. Tomando en cuenta sólo a los países en donde al inglés se le ha dado una posición administrativa especial, la población total es de más de 2 billones, o sea, más de un tercio de la población mundial. Un mercado de tal magnitud es claramente atractivo.

Por supuesto esta motivación por el negocio y la ganancia raramente se muestra tan claramente. Al contrario, el inglés es propuesto como una forma altruista de ayudar a los países menos desarrollados para que lleguen a formar parte de la actividad empresarial a fin de mejorar su condición. Pero tal como Noam Chomsky, un distinguido lingüista, señaló hace algunos años, es el sector empresarial el que efectivamente dirige a las sociedades capitalistas modernas y el compromiso fundamental de este sector es el de utilizar el mercado global de tal forma que le proporcione la ganancia máxima, pero que el mismo 
tiempo "presente su compromiso como si estuviera guiado por una profunda preocupación por el bienestar humano" (Chomsky, 1982).

\section{El imperialismo lingüístico: La perspectiva central}

El diccionario Longman define el imperialismo como: "La creencia de que es bueno hacer o agrandar los imperios, especialmente el imperio de la propia nación de uno"; y una segunda definición, despectiva, dice: "La ganancia de ventajas políticas y de comercio sobre las naciones pobres por un país poderoso que los dirige o les ayuda con dinero". El diccionario Collins Cobuild da solamente una definición para el imperialismo, y es más parecida a la definición despectiva de Longman, pero aún más rigurosa: "Un sistema de dominio, o una creencia en un sistema de dominio, en el que un país rico y poderoso controla a otros países para hacerse más rico y más poderoso".

El amplio tema de un libro titulado Imperialismo lingüistico (Phillipson, 1992) es que el idioma inglés y el negocio de la enseñanza del idioma inglés alrededor del mundo perpetúan un sistema de neoimperialismo, en el sentido que apoyan un sistema global del comercio mundial que le da ventaja a los países centrales (ricos y poderosos) y desventajas a los periféricos (países más pobres). La teoría de Phillipson del imperialismo lingüístico está modelada en la de Galtung (1980) quien describe al imperialismo en tres etapas. Primero, los colonizadores de los países del Centro viven en y dominan a los países periféricos. Segundo, se prepara una élite local en los países periféricos, quienes hablan el idioma de los colonizadores y quienes frecuentemente son educados en el país colonial. En esta etapa, en el país periférico se les da rienda suelta a las compañías multinacionales, subyugando efectivamente la economía. Tercero, la presencia del personal del centro ya no es necesaria, puesto que el control internacional puede efectivamente ser asegurado a través de los medios de comunicación y de la tecnología, especialmente tecnología computarizada.

Phillipson demuestra como el imperialismo lingüístico sigue las fases esbozadas por Galtung. Primero, la presencia de los colonizadores y la imposición de su lenguaje. Segundo, la formación de la élite local, competente en el lenguaje del colonizador y actuando como un puente político y comercial. Tercero, la etapa de la persuasión ideológica a través del uso de los medios y de la tecnología. 
Phillipson ha desarrollado su teoría del imperialismo lingüístico dentro de dos paradigmas a los que llama "el paradigma de la difusión del inglés" y "el paradigma de la ecología del lenguaje".

"El primero se caracteriza por el capitalismo triunfal, su ciencia y su tecnología y una visión monolingüe de la modernización y de la internacionalización. El paradigma de la ecología del lenguaje implica la construcción de una diversidad lingüística mundial, la promoción del multilingüismo y el aprendizaje de un idioma extranjero y la concesión de derechos humanos lingüísticos a los hablantes de todos los idiomas." (Phillipson and Skutnabb-Kangas, 1996).

En esta misma línea encontramos a otro lingüista, Alistair Pennycook, quien cuestiona la aceptación pasiva del "inglés internacional", como si fuera parte del orden natural de las cosas, señalando las fuerzas económicas y políticas del capitalismo internacional que lo han impuesto, y que al hacerlo han dominado también a las culturas. Él toma prestado el término "orientalismo" de Edward Said, a fin de señalar las políticas a favor de la educación en inglés. Él ve la mano destructiva colonialista, que fomenta tanto el anglicismo al imponer el inglés, como al orientalismo, al entorpecer efectivamente el acceso a la lengua inglesa para todos, menos para los más privilegiados. Haciendo referencia a los casos de Malasia y Singapur, Pennycook demuestra cómo el idioma inglés se ha convertido en "el distribuidor de la desigualdad" (Pennycook, 1994: 255).

Por supuesto que no falta quien critique a Phillipson y Pennycook. Una crítica ampliamente diversa sobre el tema del imperialismo lingüístico viene de Fishman (1996), quien cuestiona si la expansión continua del inglés "está de alguna forma directamente orquestada y promovida por el mundo anglófono, o si está puesto al servicio del mismo, de una manera explotadora". Sugiere que una razón más simple y menos conspiradora puede ser que, "si el negocio de los Estados Unidos es hacer negocio" y si los negocios de ese país se conducen en inglés - y casi exclusivamente es así, alrededor del mundo-, entonces el poder de la economía de EE.UU. puede por sí mismo convertirse en una punta de lanza y en un sistema de apoyo continuo para la difusión mundial del inglés" (Fishman et al., 1996:4)

Tanto Phillipson y Pennycook, como Fishman comentan sobre el tema del imperialismo lingüístico desde una perspectiva del centro (o 
el Norte, como algunos prefieren). ¿Cómo ven las cosas los sociolingüistas y los especialistas en la enseñanza del lenguaje desde los países periféricos?

\section{El imperialismo lingüístico: La perspectiva periférica}

La tesis de Phillipson de una conspiración occidental para dominar a los países del tercer mundo a través del idioma inglés es examinada críticamente por un especialista de la periferia, Suresh Canagarajah. Mientras que él acepta que "con el tipo de evidencia que Phillipson reúne, es difícil no ver teorías de conspiración en las actividades de estas agencias culturales a las que nosotros en el tercer mundo hemos hasta ahora considerado que promueven de forma altruista el lenguaje y la cultura". Este autor pasa a advertir que "sin una atención adecua$\mathrm{d}$ a a las actitudes en conflicto y a las motivaciones de los estudiantes de inglés del tercer mundo, Phillipson corre el riego de exagerar las intenciones imperialistas". (Canagarajah, 1996: 406).

También los lingüistas del continente africano critican la tesis de Phillipson. Al escribir en el contexto de Nigeria, Bisong (1995) argumenta que Phillipson ha malinterpretado la situación nigeriana. Muestra como, aún cuando el inglés se ha convertido en el idioma oficial de Nigeria, no ha tenido éxito en desplazar a los idiomas indígenas. Él sostiene que se aprende el inglés por razones deliberadamente pragmáticas y que los que lo aprenden así no son en ninguna forma víctimas del imperialismo lingüístico. Bisong concluye:

Los argumentos que conllevan la implicación de que los usuarios de este idioma no conocen lo que es mejor para sus intereses, no deberian ser simplemente vistos como condescendientes. Ellos revelan una falla monolingüe para concebir la compleja naturaleza de una sociedad multilingüe y multicultural. (Bisong, 1995: 131).

En el contexto de la planificación del lenguaje para la nueva Sudáfrica, Desai (1995) también establece este punto, mostrando como el Inglés tiene que ser visto en el contexto de un multilingüismo que es la verdadera "lengua franca" de África - una situación muy diferente a la fórmula de la mayoría de los países occidentales de una sola lengua dominante-. De nuevo, en Sudáfrica, la población no está escogiendo el inglés por un servilismo cultural - Mandela ha contribuido a erradicar esto- sino por razones prácticas, de mayor oportunidad para la próxima generación en el mercado global. 
Hay algún terreno común entre las varias opiniones de los lingüistas del centro/Norte y de la periferia/Sur, sobre la naturaleza del imperialismo lingüístico. Las ideas radicales parecen ser: en la fase temprana del colonialismo, compulsión y coerción; en la segunda, manipulación y compromiso astuto; y en la tercera fase, control encubierto a través de los medios y de la tecnología.

Aplicando este análisis teórico a una situación concreta, ahora me gustaría usar estos elementos principales del imperialismo lingüístico en sus diferentes fases, como un marco de referencia dentro del cual considerar algunos eventos significativos en la historia del idioma inglés en Hong Kong.

\section{La historia del idioma inglés en Hong Kong}

La historia del idioma inglés en Hong Kong abarca un período de aproximadamente un siglo y medio. Primero, bosquejaré brevemente los primeros días de las escuelas misioneras y de las primeras dos universidades en Hong Kong. Luego, consideraré en más detalle tres ejemplos en la historia reciente de la educación en Hong Kong: Primero la política gubernamental poscolonial sobre los modos de instrucción; segundo, un esquema para importar un gran número de profesores, hablantes nativos del inglés, como un esfuerzo por mejorar los estándares; y tercero la imposición de un examen calificador para los/las profesores de idiomas en las escuelas. Veremos hasta que punto el cargo de imperialismo lingüístico puede justamente ser presentado contra el gobierno de Hong Kong.

\subsection{Las escuelas misioneras y la escuela central}

En 1843 se abrió la primera escuela en Hong Kong. Fue fundada en memoria de Robert Morrison, el primer misionero protestante en China. Los otros grupos religiosos misioneros, los católicos y los anglicanos llegaron poco después. Las primeras escuelas tenían claramente una motivación religiosa, su objetivo principal era el de convertir a los chinos al cristianismo y a la vez se le dio prioridad a la enseñanza del inglés. También crecieron y se amalgamaron a la escuela central, otras escuelas no misioneras. La enseñanza se llevaba a cabo en chino y el currículum estaba compuesto por los clásicos chinos, la Biblia, pero el énfasis también se le daba a la enseñanza del inglés. 
El director, originalmente un misionero, era principalmente un educador y gradualmente suprimió la enseñanza bíblica, declarando que no debería ser parte de las políticas gubernamentales la conversión de los chinos.

La escuela central pronto entró en dificultades con el gobernador británico, preocupado por el pobre nivel del inglés, puesto que la escuela había sido fundada con el propósito explícito de dedicarle más tiempo a la enseñanza de ese idioma. El problema para todas las escuelas radicaba en el hecho de que tan pronto como el alumnado había aprendido un poco de inglés, dejaba la escuela para hacer negocios. Es decir, la gente en Hong Kong, veía -y sigue haciéndolo- a la educación como una preparación esencial para hacer mejorar los negocios.

Sería una mala lectura de los hechos decir que el imperialismo lingüístico era evidente en estos tempranos días de la educación en Hong Kong. Ante todo, inicialmente no era necesario tener una población local que hablara inglés. Del servicio civil se encargaba totalmente Gran Bretaña a través de sus burócratas y no había virtualmente ninguna mezcla entre la comunidad británica colonial con los habitantes locales. También muchos de los primeros misioneros sintieron que era parte de su tarea aprender chino y los hicieron muy bien.

Además, con el desarrollo de la colonia y la creciente necesidad del inglés, los padres buscaron la oportunidad de hacer que sus hijos aprendieran el idioma. No fue impuesto por un gobierno autocrático. De ahí que aún cuando un obispo llegó a ser el superintendente de las escuelas, y cambió el plan de estudios a uno mucho más cristiano, con medio día dedicado a las Escrituras, los alumnos siguieron muy entusiasmados en ingresar a la escuela anglicana, simplemente por la ventaja económica de aprender el inglés. Así, desde temprano en la historia de Hong Kong, es importante notar que los padres le dan una enorme importancia a la oportunidad de que sus hijos aprendan inglés.

Si las marcas de ley del imperialismo lingüístico son la coacción, la manipulación y el control encubierto, difícilmente se puede decir que tal situación existiera en esos tempranos días de la educación anglófona en Hong Kong. De hecho, no había coacción. La gente era libre de asistir a la escuela que quisiera. Sin embargo, tal vez se podría decir que hubo cierto grado de manipulación en las escuelas misioneras, es 
decir que usaron el anzuelo del inglés para ganar conversos al cristianismo. Y también es cierto que, al desarrollarse Hong Kong y crecer la necesidad por un cuerpo de administradores bilingües y de intermediarios de negocios, entonces la acusación del imperialismo lingüístico, en la forma de manipulación y control encubierto, podría ser más justificable.

\subsection{La fundación de la Universidad de Hong Kong y de la Univer- sidad China}

Es interesante que cuando la Universidad de Hong Kong se fundó en 1912, se tomó la decisión de que el medio de instrucción debía ser el inglés. Esto no fue muy bien recibido por los negociantes británicos de Hong Kong. Sintieron que había peligro de que si la Universidad producía un cuerpo de hombres y mujeres de negocios listos, con buen inglés, ellos podrían representar una amenaza a los intereses del comercio británico. Algunos han citado esto como un ejemplo descarado de imperialismo lingüístico - la imposición del lenguaje colonial sobre la única institución terciaria de Hong Kong-. Pero probablemente es más justo decir que la intención del gobierno británico de entonces realmente era completamente práctica, es decir, la de equipar al servicio civil con un cuerpo de personas bilingües y bien preparadas.

Más cuestionable que el caso de la institución en la que trabajo fue el caso de la Universidad China de Hong Kong, que fue fundada en 1963. El propósito de esta fue aparentemente el de proveer educación terciaria en chino, principalmente para los alumnos más pobres de las escuelas secundarias chinas. Sin embargo, en la práctica, puesto que el examen de admisión era parcialmente sobre la competencia en inglés, los alumnos de los bachilleratos que usaban el inglés como medio de enseñanza tenían una marcada ventaja. Por lo tanto, la fundación de la Universidad China sirvió realmente para fortalecer la posición de la educación que usaba el inglés como medio de enseñanza a expensas de la educación en la lengua materna.

\subsection{El medio de instrucción en la escuelas}

En Hong Kong, el medio de instrucción en las escuelas ha estado en debate por más de un siglo. Llegó a su punto máximo en 1998, después del retorno de Hong Kong a China, cuando el nuevo gobier- 
no (SAR) de Hong Kong cambió el lenguaje de instrucción en todas las escuelas secundarias del inglés al chino, exceptuando las escuelas exentas de esta regulación.

Antes de 1998, las clases en las escuelas secundarias eran impartidas en chino o en inglés, dependiendo principalmente de la opción del director y de la junta directiva. El número de escuelas en chino había descendido dramáticamente en la última mitad del siglo Xx. En 1960, $42 \%$ de las escuelas utilizaban el chino y $58 \%$ el inglés. Para 1990, unos cuantos años antes de la entrega de Hong Kong a China, solamente había un $8 \%$ en chino, y $92 \%$ en inglés.

Es difícil saber si el culpable de la falta de solución sobre el asunto del cambio del inglés al chino fue el gobierno colonial. Se puede argumentar que no había mucho que el Departamento de Educación pudiera hacer, dada la clara preferencia de los padres por la enseñanza en inglés. Una interpretación más pesimista de la acción del gobierno colonial, o más bien, de su falta de acción, sería que su negativa a actuar decisivamente sobre la pregunta del medio de instrucción fue deliberadamente calculada y que especialmente hacia el final de la tenencia del gobierno colonial, este estuvo francamente poco dispuesto a encarar el problema que resultaría en un cambio obligatorio a la enseñanza en chino. Bajo esta interpretación, estaría justificado decir que el imperialismo lingüístico del tipo clásico, colonial astutamente manipulador ha existido en Hong Kong por más de un siglo.

Pero, ipuede la acusación de imperialismo lingüístico ser hecha solo al gobierno colonial? Poco después de la entrega, el gobierno SAR estipuló la enseñanza en chino y una tormenta de protestas seguida por enfurecidas cartas a la prensa abundaron. Los estudiantes y los padres se fueron a las calles. El alumnado de ciertas escuelas prestigiosas que habían sido forzadas a cambiarse a la enseñanza en chino, amenazó con llevar al gobierno a la corte en relación con este asunto.

El editorial en el principal periódico matutino de Hong Kong, el South China Morning Post, no se equivocó al advertir:

Algunos asuntos todavía alteran los nervios. La enseñanza de la lengua materna probablemente seguirá siendo un tema contencioso por muchos años, a pesar de los obvios beneficios de permitirle a los niños aprender en el lenguaje con el que se sienten más cómodos. Cualquier cambio que afecte los planes de los padres de mandar a sus hijos a las 
escuelas preferidas con enseñanza en inglés está destinado a causar un

furor. (Editorial, SCMP, 2000)

Como resultado del cambio de política, algunas familias decidieron dejar Hong Kong e irse a otra parte. Una madre escribió una carta de despedida a la prensa, explicando que sus razones para irse eran: primero, el cambio de política gubernamental sobre el medio de instrucción; segundo, que ella quería que sus hijos "crecieran en un ambiente internacional en contacto con el inglés, cuya fluidez es esencial para prospectos laborales"; y tercero, porque en Hong Kong, "por una noción equivocada de patriotismo, el inglés es considerado un lenguaje colonial y casi todos los departamentos gubernamentales conversan sólo en chino." En consecuencia, ella finalizó: "Me voy para Singapur con mi familia. Buena suerte a Hong Kong cuando sea una colonia china." (Wu, 1998).

\subsection{El Proyecto de los profesores nativos de inglés}

El Proyecto de los Profesores Nativos de Inglés (NETS, por sus siglas en ese idioma), consistía en importar a profesores nativos del inglés para las escuelas de Hong Kong. El plan era el de proveer a cada escuela Secundaria con dos o tres profesores expatriados. El Concilio Británico hizo el reclutamiento.

El criterio para definir quién era el hablante nativo era el país de origen de la persona y no su competencia en inglés para la comunicación internacional. Este enfoque postergó la revaloración actual de la noción del hablante nativo. En un mundo cada vez más dinámico, el país de nacimiento es solamente un factor, entre otros que debería ser considerado - factores como la competencia, la fluidez, la inteligibilidad internacional (en la que algunos hablantes nativos obtienen un puntaje bajo), uso como un idioma dominante, e identificación cultural-.

Es más, la obvia ventaja de que disfrutaba un profesor de inglés local, en lo que respecta a la identidad étnica y cultural compartida, debería ser considerada entre los factores más apropiados, al escoger a los profesores de inglés en un país en donde el inglés no es el idioma nativo. Chen Eoyang, habitante de Singapur, condena "el mito del experto nativo", el que sataniza la enseñanza de cualquier idioma para los que nos son hablantes nativos, ya sea el francés, el chino o el 
inglés. Él reconoce que algunos hablantes nativos del inglés pueden ser excelentes profesores de idiomas. No obstante, agrega: "Insistiré en que ser un nativo de un idioma no nos califica automáticamente para ser un profesor de idiomas efectivo." (Chen Eoyang, 1999: 19)

El presente plan NET, como su predecesor, se ha encontrado con hostilidad de parte de los profesores de inglés locales de Hong Kong, quienes se oponen a las condiciones preferenciales y a las mejores prestaciones dadas a los profesores extranjeros, y quienes sienten que los extranjeros, con su falta de conocimiento del idioma local, de la cultura y del sistema educativo, son menos eficientes que muchos de los profesores locales.

En relación con el imperialismo lingüístico, no parece que el plan NET merezca una etiqueta. A los profesores locales se les está haciendo sentir inadecuados e inferiores, al compararlos con los profesores extranjeros, que justifican sus posiciones privilegiadas por el simple hecho de ser hablantes nativos del inglés.

\subsection{El examen de calificación para los profesores de inglés de Hong Kong}

Si el cambio al chino como medio de instrucción y el plan NET causaron polémica, mucho más controversial fue el anuncio hecho por el gobierno de un examen de calificación para todos los profesores de inglés (totalizando cerca de 14,000 personas). Esta propuesta fue recibida con indignación por la gremial de Profesores Profesionales, la que afirmaba que el gobierno estaba haciendo de los profesores un chivo expiatorio y culpándolos por el pobre estándar del inglés en Hong Kong, cuando en realidad otros factores más poderosos, como el cambio de ambiente para la enseñanza del inglés en Hong Kong, la baja motivación estudiantil, la motivación más baja del estudiante, la falta de profesores de inglés calificados, etc., eran los culpables.

Una parte principal del problema era que cerca del $25 \%$ de los profesores de inglés en las escuelas secundarias y casi el $50 \%$ de los profesores en la primaria no estaban calificados para la enseñanza del inglés. Ellos impartían el inglés porque los directores de las escuelas se los pedían, debido a la falta de profesores de inglés calificados. Esta indignación de esos profesores al exigirles que tomaran el examen en inglés fue por lo tanto entendible. De igual forma, los que habían 
calificado en inglés se rehusaban a tomar el examen, preguntando para que sirvió su previa calificación si se les pedía tomar un examen adicional para probar su competencia en inglés.

La gremial de profesores profesionales de inglés recogió firmas de 37,000 de sus miembros e hizo un llamado a que se boicoteara el examen. Al mismo tiempo hicieron una manifestación de protesta. La manifestación apareció en la primera página de la prensa, con más de 6,000 profesores marchando por las oficinas gubernamentales centrales. El presidente de la Gremial de Profesores Profesionales dijo, en un discurso provocador:

Demandamos que el Gobierno acepte nuestra petición de abolir el examen calificador tan pronto como sea posible. La cosa más importante que el Gobierno puede hacer para mejora el estándar del idioma para nuestros estudiantes es reducir el tamaño del as clases, asi como la carga de trabajo de los profesores. El gobierno no debería confundir al público para que crea que el examen calificador va a mejorar los estándares del idioma. (Wan, 2000)

Con relación al imperialismo lingüístico, a los profesores se les está haciendo sentir de nuevo inadecuados y amenazados por la posibilidad de fallar el examen y aun de ser desplazados en sus responsabilidades. Mientras tanto, las editoriales extranjeras aparecen en escena, como la Cambridge University Press, anunciando su nuevo libro Inglés para el profesor, e ilustrando "cómo coincide con los requerimientos del examen calificador."

\section{Conclusión}

Resumiendo: Esta charla consideró primero cómo el inglés se ha convertido en la lengua franca de facto del mundo. Luego analicé algunos de los rasgos del imperialismo lingüístico, desde un punto de vista central y uno periférico. Después, tomando a Hong Kong como ejemplo, esbocé algunos momentos importantes en la historia del idioma inglés en una situación colonial.

Lo que surge de esta conferencia es que, por un lado, hay una claridad de propósito en el sector comercial, en los países del centro o Norte, que claramente están felices de ver la expansión del inglés fortaleciendo su control global e incrementando sus ganancias. Por otro lado, en los países periféricos o del Sur hay una actitud ambivalente sobre el inglés. 
Lo ven como una amenaza para su cultura y para los idiomas nativos, pero al mismo tiempo lo quieren y están dispuestos a hacer grandes sacrificios para obtenerlo, al menos para sus hijos.

Es esta actitud ambivalente hacia el inglés a la que me referiré en mi próxima conferencia. En ella describiré como en partes del mundo como en China, la India, y las Filipinas, las actitudes hacia el inglés están cambiando, y la unidad y variedad lingüística son considerablemente compatibles.

\section{SEGUNDA CONFERENCIA:}

\section{EL INGLÉS COMO UN IDIOMA MUNDIAL: LAS ACTITUDES}

\section{ALREDEDOR DEL MUNDO}

En mi primera conferencia consideré la noción del imperialismo lingüístico en un sentido general. Luego la examiné más profundamente en relación con una situación particular del idioma en Hong Kong. Vimos, por una parte, cómo se adapta tan bien a los intereses del comercio internacional, que el inglés debe expandirse como un idioma global, ya que esto hace la comercialización mucho más fácil y efectiva. Por otra parte, vimos los peligros de la diversidad cultural y lingüística impuesta por la implacable expansión del inglés. Es en este segundo tema en el que deseo proseguir ahora.

Primero, consideraré como la noción de las variedades del inglés ofrece una solución parcial al dilema de la identidad individual versus la inteligibilidad mutua. Segundo, describiré los resultados de estudios en la región asiática sobre las actitudes cambiantes hacia el idioma inglés, especialmente entre los jóvenes. Particularmente, veré tres países con los que tengo alguna familiaridad, a saber: China, India y las Filipinas. En cada uno de estos tres países un trilingüismo efectivo ha emergido como la norma lingüística. Tercero, consideraré los efectos potencialmente destructivos del monolingüismo del inglés sobre otros idiomas y culturas, y haré un llamado a la preservación de la diversidad lingüística..

\section{El inglés mundial y los ingleses mundiales}

En los círculos académicos, las actitudes han cambiado hacia las ideas del inglés mundial, especialmente en las últimas décadas. En 1981 
se fundó una publicación para los profesores de inglés llamada World Language English ("El inglés, idioma mundial"). Los primeros números de esta publicación muestran la modernidad en el enfoque de su editor, el doctor Bill Lee, hacia el inglés como un idioma mundial:

El inglés es, abora sobre todo, un idioma internacional, usado o entendido en la mayor parte del globo y en una gran variedad de circunstancias. En el mundo actual, sirve como medio de expresión y de comunicación, no sólo entre las personas que lo han adquirido como su lengua materna, sino -en una escala mucho más marcada-entre los usuarios no nativos, cuyas lenguas maternas son muchas y variadas. El inglés como un idioma mundial no 'pertenece' solamente a los hablantes de la lengua materna, sino a cuantos bacen uso efectivo de este. (Lee, 1981:1)

Desgraciadamente, la publicación de Lee circuló solamente por cuatro años. Sin embargo, fue reemplazada por otra publicación, llamada significativamente World Englishes ("Los ingleses mundiales"). Esta publicación, con su título deliberadamente desafiante, era una colección de artículos sobre el inglés en sus formas en desarrollo y sus variantes nacionales, cada una con su respectivo derecho a un lugar en la mesa del inglés. Su editor buscó con vigor y entusiasmo la noción de los ingleses mundiales, en plural:

Cualquiera que sean las preocupaciones de los puristas, me parece que bay mucho que celebrar en la expansión del inglés como un idioma mundial. En donde más de 650 idiomas artificiales ban fallado, el inglés ba tenido éxito; en donde muchos otros idiomas naturales con poder político y económico para respaldarlos han fallado, el inglés lo ba logrado. Una razón para esta supremacia del inglés es su propensión a adquirir identidades nuevas, su poder de asimilación, su adaptabilidad a 'descolonizarse' como un lenguaje, su manifestación en una gama de variedades, y sobre todo, su idoneidad como un medio flexible para la creatividad literaria $y$ otros tipos de creatividad a través de los idiomas y las culturas. (Kachru, 1988: 8).

Sin embargo, esta noción que se tuvo de muchas variedades aceptables del inglés tuvo sus propias dificultades:

El surgimiento de una forma del inglés recién identificada trae consigo problemas como el de su idoneidad para usarse como modelo. Si "el inglés nigeriano refinado" existe como una entidad identificable $y$ 
reconocible, ¿debería esa forma de convertirse en el modelo y el objetivo usado en la enseñanza del inglés en las escuelas de Nigeria? Si no se utiliza una forma local, iqué actitud deberian adoptar los profesores hacia ella, y cómo podrian y deberian justificar el uso de un modelo derivado de cualquier otra parte? (Stevens, 1981: 4).

El debate sobre las variedades en contra del inglés estandarizado comenzó en los ochenta, cuando Randolph Quirk, recomendó el inglés británico estándar como la norma de enseñanza, mientras que Braj Kachru defendía la causa de las variedades. Quirk sostenía: "La gama relativamente estrecha de los propósitos para los cuales el hablante no nativo necesita hablar el inglés es discutiblemente bien provista por una sola forma monocromática estándar, que se vea tan bien en el papel como suena en el habla." (Quirk and Widdowson, 1985: 6). Mientras que Kachru creía que: "entonces, lo que se necesita es cambiar de un autoritarismo lingüístico de la variedad de "lo que el hablante nativo dice", a un realismo de la comunidad hablante, con la comprensión de que la exclusividad funcional y sociocultural de cada confraternidad del habla (speech-fellowship) no se puede impedir." (Quirk and Widdowson, 1985: 25).

El debate continuó hasta los noventa fecha en que Quirk (1990), designó el enfoque de las variedades como lingüística de la liberación". Mientras Kachru, cuestionó "las estrategias que usan los hablantes nativos con influencia y poder para controlar la dirección del inglés, sus innovaciones, y su aculturación" (Kachru, 1991: 9).

Otros especialistas en lingüística aplicada y en la enseñanza del inglés apoyaron a Kachru por considerar fútil el esfuerzo para mercadear solamente al inglés británico estándar. Widdowson (1994) rechazó la noción de que el inglés de pronunciación recibida, RP (por sus siglas en inglés. El RP es ahora un dialecto del inglés de las minorías, aún en el Reino Unido), debería ser la norma central para la enseñanza del inglés alrededor del mundo. El autor señalaba el peligro de tratar al inglés como un artículo comercial, poseído por "nosotros" para serle vendido a "ellos", o, al menos, a esos que puedan pagarlo. Widdowson aceptó la existencia de las variedades del inglés alrededor del mundo como un hecho establecido y, recomendó que en lugar de tratar de forzar el inglés británico estándar en todos, se le debería prestar atención al desafío más urgente de lograr una inteligibilidad mutua entre las diferentes variaciones nacionales del inglés.

Dos conferencias sobre la hegemonia cultural de la lengua inglesa 
Cuando nos acercábamos al milenio, la noción de "inteligibilidad internacional" era la clave de la posición comprometida de David Cristal, lingüista altamente respetado:

Una filosofía de la diversidad, que reconozca la importancia de la bibridización, evidentemente, no excluye la noción de un estándar. Por el contrario, la necesidad de mantener la inteligibilidad internacional demanda el reconocimiento de una variedad estándar del inglés, al mismo tiempo que la necesidad de mantener la identidad local demanda el reconocimiento de las variedades locales del inglés. Mi principio fundamental es que necesitamos de ambos, en un mundo lingüisticamente saludable. $Y$ nuestros modelos, tanto teóricos como pedagógicos, necesitan permitir la complementaridad de estas dos funciones del lenguaje. (Cristal, 1999: 15).

Otra figura importante en el continuo debate fue Tom McArthur, el editor de otra publicación en el área, llamada English Today, la que, tras un período de tiempo, tuvo artículos excelentes sobre el desarrollo del inglés en el mundo moderno. En este libro, llamado intencionadamente The English Languages ("Los idiomas ingleses"), McArthur emfatiza la diversidad del inglés moderno:

En el mundo, miles de millones de personas usan el inglés diariamente, pero no muchos pueden tener éxito para entenderse entre ellos. $A$ pesar del éxito de su forma (o formas) estandarizadas en muchos paises, el complejo llamado 'Inglés' es inmensamente diverso - probablemente más diverso de lo que cualquier otro idioma lo halla sido jamás-y es muy probable que llegue a ser todavia más diverso en el próximo siglo. (McArthur, 1998, prólogo).

Al preguntarle un periodista de Newsweek, “ ¿Háurá algún espacio para un estándar globalmente reconocido para el inglés hablado?”, McArthur respondió:

No existe tal estándar, y dudo que jamás lo halla. El inglés es un poco como un pulpo. Sus tentáculos van a todas partes, y nuevas clases de personas lo toman. No hay una autoridad central como la Academia Francesa. Debido a que es tan dominante, pero al mismo tiempo tan variado, el inglés puede ser atractivo y peligroso - peligroso porque ejerce un poder enorme-. (McArthur, 1993: 56).

El verdadero significado de la noción de las variedades del inglés radica en su habilidad para ofrecer un camino medio entre la indivi- 
dualidad lingüística y la inteligibilidad mutua. El inglés es un idioma global, pero sus hablantes de diferentes países y regiones pueden preservar cierto grado de individualidad al usar su propia forma del inglés, una forma que es distintiva, pero también inteligible para otros angloparlantes alrededor del mundo.

Parte del debate sobre el inglés estándar versus las variedades, es si estas últimas pudiesen llegar a ser mutuamente ininteligibles, e incluso, que puedan volverse idiomas diferentes, de igual forma como el latín se fragmentó en francés, italiano, español y portugués. Aunque uno nunca puede estar seguro, esto parece imposible, y es significativo el que las predicciones de los filólogo Noah Webster en 1789, y Henry Sweet, un siglo más tarde, en 1977, de que los ingleses británicos y americanos llegarian a ser mutualmente ininteligibles, ha demostrado ser incorrecta.

\section{Actitudes cambiantes hacia el inglés: China}

Las actitudes existentes en China hacia el inglés han variado a través de la historia, y este idioma ha sido acogido, bien con agrado, bien de una manera negativa, dependiendo de la situación política prevaleciente en China en el momento. Originalmente, los chinos no sintieron ninguna necesidad del Occidente y deliberadamente evitaron todo contacto, por temor de la contaminación cultural. En 1949, por ejemplo, el año de fundación de la República del Pueblo de China, el Ministro de Educación emitió un "Plan para la Instrucción del Inglés en las Escuelas Secundarias", en el que la meta del aprendizaje del idioma inglés fue claramente establecida para servir a la Nueva República, en donde no se permitía ningún texto que contuviera "pensamiento capitalista". En el currículo, el lugar del inglés lo ocupó el ruso, y para 1954, el ruso se había convertido en el único idioma extranjero que se enseñaba en la escuelas chinas.

Al principio de los años sesenta, el inglés entró en escena de nuevo, puesto que la necesidad de China de abrirse al escenario internacional era más evidente. Sin embargo, el péndulo se balanceó de nuevo para otro lado con la Revolución Cultural, desde mediados de los $60 \mathrm{~s}$ hasta mediados de los setenta, cuando el inglés fue prohibido en las escuelas, y donde se tildaba a los profesores de idiomas extranjeros como espías. Otro giro del péndulo hizo que en 1978 el Ministro de 
Educación restableciera el inglés en los programas de estudio, dándole de nuevo prominencia en las escuelas, a la par del estudio del chino y las matemáticas. Para principio de los ochenta, se había convertido en una materia obligatoria para el examen de ingreso universitario.

Como nota personal, una de la experiencias de enseñanza más memorables de mi vida fue un grupo de clase que tuve en Inglaterra, formado por doce de los primeros alumnos a quienes se les permitió salir de China después de la revolución cultural. Ellos fueron los estudiantes más inteligentes y aplicados que he tenido, cada uno dedicado a estudios de doctorado en Gran Bretaña. Ellos estaban en el laboratorio de idiomas desde las 6.00 a.m. y me mantenían hasta entrada la noche para practicar la conversación, por un periodo de seis semanas. Ellos adquirieron una competencia sorprendente tanto en el inglés escrito como en el hablado.

En la actualidad, el entusiasmo por aprender inglés en toda China es increíble. No existe el sentimiento de que podrían estar perdiendo algo de su propia identidad cultural al aprender el Inglés. El Reporte de la Conferencia "Inglés 2000" en Pekín, organizada juntamente por el Concilio Británico y la Comisión de Educación del Estado de China, describe la motivación de los chinos al aprender inglés: "Lo aprenden porque es el lenguaje de la ciencia y de la mayoría de publicaciones de investigación; porque es el idioma neutral del comercio, el uso estándar de los viajes internacionales y de la comunicación. Lo aprenden porque se encuentra más software en inglés que en todos los otros idiomas juntos".

Muchos estudios, elaborados durante los últimos treinta años, han revelado el marcado cambio de actitudes hacia el inglés, especialmente entre los jóvenes. En un estudio de 1975 (Fu, 1975) se examinó a los estudiantes de secundaria sobre sus actitudes para el inglés y sus hablantes nativos.. Se encontró un alto grado de acuerdo sobre la importancia del inglés como un idioma internacional y como modo de obtener un trabajo ( $84 \%$ de los examinados). Sin embargo, hubo renuencia a darle demasiada prominencia al inglés: $57 \%$ de las personas encuestadas afirmó que el inglés debería ser opcional, en lugar de ser una materia obligatoria en la escuelas, mientras que el $69 \%$ aseveró que ellos se sentirían incómodos si un profesor chino les hablara inglés a ellos. Al preguntarles sobre sus sentimientos por los hablantes nativos del inglés, muchos se rehusaron a contestar, mientras que más 
de la otra mitad de los que contestaron fucron bastante críticos. Ellos describieron a los occidentales en términos poco halagadores, tales como arrogantes, orgullosos, despreciativos, astutos, fríos, desconsiderados para con otros, rudos y racistas, "que desprecian a los chinos y a quienes no hablan el inglés".

Otro estudio (Person, Fu y Lee, 1980), que les pedía a los estudiantes responder sobre diferentes declaraciones sobre el uso del inglés, encontró resultados similarmente ambivalentes, con el inglés visto como importante, pero también como una amenaza para la identidad china de los sujetos. Mientras que la pregunta " ¿Desearía poder hablar un inglés fluido?" recibió una respuesta positiva sorprendente, de igual manera sucedió con otras dos interrogantes: "Si uso el inglés, ¿significa que no soy patriota?" y "¿Cuando hablo inglés, siento que ya no sigo siendo chino?"

Sin embargo, al aplicar estos estudios preliminares fueron replicados en 1990 (Pennington y Yu, 1994), hubo notables diferencias en los resultados. Mientras que todavía había algún acuerdo sobre la importancia del inglés para conseguir un trabajo, hubo un cambio notable en las actitudes ante la posible pérdida de identidad étnica a través del uso del inglés. Estas declaraciones "Si uso el inglés significa que no soy patriota" y "Cuando uso el inglés, siento que ya no sigo siendo chino, ahora recibieron un fuerte desacuerdo.

Un estudio, el cual conduje yo mismo, más tarde en 1990, cubría tanto a gente trabajadora joven como a estudiantes. Estas actitudes encontradas hacia el inglés fueron considerablemente pragmáticas y les faltaba la predisposición cultural: Se veía al inglés simplemente como esencial para propósitos internacionales y como una herramienta necesaria para encontrar un mejor trabajo.

\section{La India}

Se cree que la primera persona de habla inglesa que visitó a la India se fue un embajador del rey Alfredo el Grande, en el año de 884 . Vino como un enviado con regalos para la tumba de Santo Tomás. El siguiente contacto registrado fue en 1600 , cuando los comerciantes establecieron las compañías de intercambio de la India Oriental, con sus fábricas en Madrás, Bombay y Calcuta. La India fue considerada por las compañías de intercambio en esos primeros días como un mercado 
atractivo, de igual forma en que por las compañías multinacionales ven a China actualmente.

Phillipson (1992: 110), el autor de un libro significativo sobre el imperialismo lingüístico, observa: "La importancia del idioma fue comprendida desde la fase temprana de la expansión del imperialismo. En la India, el idioma inglés fue visto como una fuerza para la 'modernización' del país, que podría crear un mercado listo para los productos británicos".

Raras veces se puede encontrar una declaración más severa que la de la carta del noble Macaulay, fechada en 1835. En la misiva, Macaulay asevera, como un hecho más que evidente, "que vale más la pena conocer el inglés que el sánscrito o el árabe" y prosigue afirmando que "es imposible hacer de los nativos de todo este país buenos estudiantes de inglés". Él resume el objetivo de la educación en la India como el de "formar una clase de individuos que puedan ser intérpretes entre nosotros y los millones a quienes gobernamos, una clase de personas, indios de sangre y color, pero ingleses en gustos, opiniones, en moral y en intelecto".

Como observa el lingüista de Sri Lanka, Wimal Dissanayake: "Cuando el inglés se convirtió en el lenguaje de la administración, el comercio, la educación, el derecho y la cultura, se vio el constante deterioro y la marginación de las prácticas discursivas tradicionales. Fue la intención no declarada de los colonizadores el domesticar a la sociedad conquistada, a través de prácticas occidentales significativas, codificarla y taxonomizarla de acuerdo a sus ideologías". (Dissanayake, 1993: 339).

Lo que hace al imperialismo lingüístico de la India particularmente molesto es que la herencia cultural de ese país es inmensamente rica y antigua. Solamente el la familia lingüística draviniana, la mayoría de cuyos integrantes se encuentran en el sur y el oriente de la India, incluye al tamil, que tiene registros escritos que datan del tercer siglo antes de Cristo.

¿Cuál es la actitud de la India moderna hacia el inglés? Sorprendentemente, hay muy pocos rasgos de amargura y resentimiento. Particularmente entre los jóvenes, el inglés es visto simplemente como un medio apropiado de comunicación internacional, al cual están felices de adoptar en su propia familia de lenguajes, en una forma que es muy distintivamente india, pero aún fácilmente inteligible para otros hablantes internacionales del inglés. 
Como lo pone Salman Rushdie: "El debate sobre la propiedad o idoneidad del inglés en la India posbritánica ha sido tenso aún desde 1947; pero ahora, Yo encuentro, que es un debate que tiene sentido sólo para la generación más vieja. Los niños de la India independiente no parecen ver al inglés como irremisiblemente impregnado de su procedencia colonial. Lo usan como un idioma indio, como una de las herramientas que tienen que transmitir". (citado en Cristal, 1997. 136). Se estima que hay alrededor de 50 millones de indios que usan el inglés regularmente, con un alto nivel de competencia y fluidez. Este es más que el número de hablantes nativos del inglés en Gran Bretaña. Cabe hacer notar, que el inglés de algunos de ellos es menos inteligible a nivel internacional que el de los hablantes de inglés de la India.

\section{Las Filipinas}

Las Filipinas fueron una colonia de España durante aproximadamente trescientos años y después, de los Estados Unidos, por un período más corto. Ahora, los jóvenes rara vez hablan el español en las Filipinas, mientras que todas las clases sociales hablan el inglés (con un acento estadounidense). Cuando el país ganó su independencia en 1946, se comenzó un movimiento para popularizar el filipino como lenguaje nacional. Este es una forma estandarizada del tagalo, el idioma indígena de la isla central de Luzón, donde se encuentra Manila, la capital. Las Filipinas, un país de miles de islas separadas, tiene una rica variedad lingüística, con idiomas como el cebuano, en el sur, idioma que hablan más de 10 millones de personas y el ilocano, en el norte, lengua en la que se expresan cerca de cinco millones de hablantes.

¿Cuál es la actitud de los jóvenes filipinos hacia el inglés en la actualidad? ¿Lo ven como un remanente del colonialismo? Desde mi experiencia, yo diría que casi nada. Aparte de una minoría de nacionalistas celosos, la mayoría de filipinos parecen demasiado contentos para aceptar la obvia influencia estadounidense de su política y su cultura. Las películas americanas y la música popular dominan, y para muchos filipinos su mayor deseo en la vida sería emigrar a los Estados Unidos.

Hace algunos años, la presión nacionalista y la erupción del Monte Pinatubo llevaron al retiro de las tropas americanas de las filipinas, pero ahora las islas son consideradas de tal importancia estratégica 
para los Estados Unidos, que la combinación de la no elegida presidenta Arroyo, fuertemente proestadounidense, y la conveniente inclusión de Abu Sayaf en la guerra del señor Bush contra el terrorismo le han posibilitado a las tropas norteamericanas, los GI Joes, regresar a las filipinas. Tiene que ser dicho, la mayoría de los filipinos parecen estar muy contentos con esto. Aunque las tropas han sido inicialmente instruidas a mantener un perfil bajo, su presencia será pronto más obvia, y serán recibidos con el usual "Hola Joe" por las chicas filipinas'.

\section{Una fórmula trilingüe}

En los tres paises considerados arriba, China, la India y las Filipinas, se ha ensayado una fórmula de planeamiento del lenguaje, con un éxito razonable, que podría convertirse en un programa para otros países del mundo. En China, el inglés se ha convertido en un idioma internacional de opción, el mandarín o el utonghua (lenguaje común) han sido designados como el idioma nacional y el idioma de vínculo por todo el país; y en cada una de las regiones de China, los lenguajes locales como el amoy y el cantonés, son preservados como lenguas maternas, los idiomas del corazón y del hogar.

En la India y las Filipinas es lo mismo, con el inglés como idioma internacional, el hindi y el tagalo como los intranacionales, esto es, idiomas de conexión, y una gran variedad de idiomas locales, como el kannada o el malayalam en la India, y el visayan o ilocano, en las Filipinas, preservados como las lenguas maternas.

Como un medio de promover la ecuanimidad de la comunicación Inter e intra nacional, mientras que al mismo tiempo se preserva la diversidad cultural y lingüística, esta fórmula trilingüe tiene mucho que recomendarle y bien podría llegar a ser la norma para muchos países en el mundo.

\section{El inglés americano}

Mientras que en el pasado la expansión del inglés fue causada principalmente por la expansión colonial británica, ahora es del dominio del comercio americano, sus medios globales, y su poder militar que son las fuerzas impulsoras en la expansión del inglés alrededor del mundo. La forma hablada del mundo del inglés, el que está emergiendo 
gradualmente ahora se parece un poco más al inglés americano estándar que al inglés de Inglaterra o a cualquier otra forma.

¿Pero qué hay del estado del inglés en la propia América? Bajo la creciente influencia del español, especialmente en el sudeste de los Estados Unidos, un movimiento lingüístico de derecha ha crecido en los estados que sienten la necesidad de establecer legalmente el inglés como el idioma nacional de los Estados Unidos. Opuesto a este enfoque defensivo, los estadounidenses liberales favorecieron el ánimo positivo del bilingüismo, y aún el multilingüismo.

La Sociedad Lingüística de América, mientras aprueba la política general de hacer del inglés el idioma común, agrega: "La promoción de nuestro propio idioma no necesita y no debería darse a costa de la violación de los derechos de las minorías lingüísticas."

También en apoyo al pluralismo lingüista, un proyecto de ley, conocido como "La resolución de más inglés", English Plus Resolution, fue presentada en la Casa de los Representantes por José Serrano en 1995. Esta enfatizaba los beneficios del multilingualismo para América y recomendaba que el gobierno de los estados unidos debía, por un lado, apoyar una política de competencia completa en Inglés para los residentes de los Estados Unidos, pero por el otro lado, deberían "conservar y desarrollar los recursos lingüísticos de la nación mediante el fomento a todos los residentes de este país a aprender y mantener habilidades en un idioma diferente del Inglés". Sin embargo, este proyecto de ley fue considerado muy extremo por el ala derecha, y no tuvo mayor éxito.

\section{El futuro, ¿monolingüe o multilingüe?}

¿Qué nos depara el futuro? Llegaran los países a convertirse gradualmente en monolingües en Inglés o será el multilingüalismo la norma? ¿Y cuales son los prospectos para la supervivencia de los reducidos idiomas minoritarios? La respuesta a la segunda pregunta es probablemente un tanto deprimente. Los idiomas que no tienen ningún poder político y económico respaldándolos están muriendo rápidamente. El lingüista Krauss (1992) piensa que es plausible que en el presente siglo veamos la muerte de $90 \%$ de los idiomas de la humanidad.

Quiero compartir una historia personal. Mi abuelo vino de las tierras altas de Escocia y hablaba escocés gaélico como idioma nativo. Su

Dos conferencias sobre la hegemonia cultural de la lengua inglesa 
hija, mi madre, hablaba un poco de gaélico, pero, sobre todo, inglés. Yo no hablo ni una palabra de aquél idioma, ni tampoco ninguno de los miembros de mi familia. Esto es cierto de casi todos mis contemporáneos cuyos abuelos hablaban gaélico. Así, en un corto tiempo de tres generaciones, el gaélico, un idioma que es mucho más viejo que el inglés, un idioma con una literatura y cultura muy ricas, prácticamente ha muerto. Si estamos un tanto disgustados por la destrucción ambiental de la vida animal y vegetal de nuestro planeta, deberíamos preocuparnos más por su degradación lingüística y cultural.

Sin embargo, existe un problema mayor que afecta tanto a la protección del ambiente como a la preservación de la diversidad lingüística. Cuestan mucho dinero, y por lo tanto, serán ampliamente controladas por los países ricos y poderosos que tienen mucho dinero, obviamente, los Estados Unidos. Tristemente, por lo tanto, las cosas no se ven tan bien, al menos si uno juzga desde el rechazo al tratado de Kioto de la actual administración Bush, sobre la base que va a dañar la economía estadounidense. Para este año que viene, alrededor de 400 billones de dólares han sido presupuestados para mantener la supremacía militar norteamericana, mientras que una mísera suma se ha destinado a mantener la diversidad lingüística y cultural. Aún así, seguramente el incrementado entendimiento cultural ofrece más prospectos para la paz mundial que la intensificación militar y el auge del comercio de armas.

Yo creo que son los países de América Latina los indicados para promover que los Estados Unidos persigan una política lingüística que vaya más allá del monolingüismo. El español es el único idioma que tiene la posibilidad de hacer una seria impresión sobre el monolingualismo americano y de mantener al menos el bilingualísmo como una meta nacional para los Estados Unidos. Si América realmente promoviera el bilingualismo nacionalmente, haria más fácil para el resto del mundo mantener el multilingualismo.

Daré la última palabra a David Cristal, una sobria advertencia al final de su excelente libro, El inglés como un idioma global: “ En 500 años, ¿será el caso que todos serán automáticamente insertados al inglés tan pronto como nazcan? Si esta es parte de una riquísima experiencia multilingue para nuestros futuros recién nacidos, esto sólo puede ser una buena cosa. Si este es para entonces el único idioma que puede ser aprendido, esto sería el más grande desastre intelectual que este planeta halla jamás conocido". 


\section{Refererencias}

Bisong, J. 1995. Language choice and cultural imperialism: A Nigerian perspective. English Language Teaching Journal 49, 2, 122-32.

Bowers, P. 1996. Report on English 2000. London: British Council.

Boyle, J. 1995. Hong Kong's educational system: English of Chinese? Language, Culture and Curriculum 8, 3, 291-304.

Boyle, J. 1997. Imperialism and the English language in Hong Kong. Joumal of Multicultural and Multilingual Development 18, 3, 169-181.

British Council Annual Reports. 1940-1990.

Canagarajah, A. S. 1996. Review of Linguistic Imperialism, R. Phillipson, in Journal of Multilingual and Multicultural Development 17, 5, 404408.

Chen Eoyang, E. 1999. English as the world's language. The Hong Kong Linguist 19, 16-22.

Cheung, M. K. 2001. Boycott the benchmark test. PTU Nerws No. 40.

Chomsky, N. 1982. Towards a New Cold War. London: Sinclair Browne.

Crystal, D. 1997. English as a Global Language. London: Cambridge University Press.

Crystal, D. 1999. The future of English. English Today 15, 2, 10-20.

Davies, A. 1996. Ironising the myth of linguicism. Journal of Multicultural and Multilingual Development 17, 6, 485-497.

Dissanayake, W. 1993. Symposium on Linguistic Imperialism. Review. Perspective 1. World Englishes 12, 3, 336-342.

Editorial. 2000. South China Morning Post September 29.

Estrada, A. 1992. Hold your tongue: bilingualism and the politics of English only. The Washington Post, 15 October.

Fishman, J., Conrad, A., Rubal-Lopaz, A. (eds.). 1996. Post-Imperial English. New York: Mouton de Gruyter.

Forestier, K. 2000. Study the costs of quality education. South China Morning Post June 18. 
Fu, G. 1979. Bilingual education in Hong Kong: a historical perspective. Working Papers in Language and Language Teaching 1, 1-10.

Galtung, J. 1980. The True Worlds: a Transnational Perspective. New York: The Free Press.

Ignatieff, M. 1995. The state of belonging. TIME Magazine, 27 February.

Kachru, B. 1988. The sacred cows of English. English Today 16, 3-8.

Kachru, B. 1991. Liberation linguistics and the Quirk concern. English Today 25, 3-12.

Krauss, M. 1992. The world's languages in crisis. Language, 68, 1, 7-8.

Lai, M. L. 1999. JET and NET: a comparison of native-speaking English teachers schemes in Japan and Hong Kong. Language, Culture and Curriculum 12, 3, 215-228.

Lander, R. 2000. Teachers perturbed by benchmark tests. Letter to South China Morning Post May 24.

Lee, W. 1981. Editorial. World Language English 1, 1, 1-2.

Llewellyn, J. 1982. A perspective on education in Hong Kong. Hong Kong: Government Printer.

Lowenberg, P. 2000. Non-native varieties and the sociopolitics of English. In The Sociopolitics of English Language Teaching, Hall, J and Eggington, W. (eds.). Clevedon: Multilingual Matters.

McArthur, T. 1993. What was that you said? Newsweek April 26.

McArthur, T. 1997. English the international language. English Today, $49,12,1$.

McArthur, T. 1998. The English Languages. Cambridge: Cambridge University Press.

Newmark, P. 2000. Translation now. The Linguist, 39, 3.

Parakrama, A. 1995. Debegemonizing Language Standards. London: Macmillan.

Pennington, M. 1998. Language in Hong Kong at Century's End. Hong Kong: Hong Kong University Press.

Pennington, M. and Yu, F. 1994. Assessing pre-1997 language attitudes in Hong Kong. Research Report, City University of Hong Kong. 
Pennycook, A. 1994. The Cultural Politics of English as an International Language. London: Longman.

Phillipson, R. 1992. Linguistic Imperialism. Oxford: Oxford University Press.

Phillipson, R. 1997. Realities and myths of linguistic imperialism. Journal of Multicultural and Multllingual Development 18, 3, 238248.

Phillipson, R. and Skutnabb-Kangas, T. 1996. English only worldwide or language ecology.

Pierson, H., Fu, G. and Lee, S.Y. 1980. An analysis of the relationship between language attitudes and English attainment. Language Learning, 30, 289-316.

TESOL Quarterly 30, 3, 429-447.

Quirk, R. 1990. Language varieties and standard language. English Today 21, 3-10.

Quirk, R. and Widdowson, H. (eds.) 1985. English in the World. London: Cambridge University Press.

Sinclair, J. 1988. Models and monuments. English Today 15, 3-6.

Strevens, P. 1981. Forms of English: an analysis of the varieties. In English for Cross-Cultural Communication,. London: Macmillan.

Tsui, A., Shum, M., Wong, C., Tse, S., Ki, L. 1999. Which agenda? Medium of instruction policy in post-1997 Hong Kong. Language, Culture and Curriculum 12, 3, 196-214.

Wan, C. 2000. 6000 Teachers march. South China Morning Post June 11. Widdowson, H. 1994. The ownership of English. TESOL Quarterly $28,2,32-48$.

Wu, G. 1988. Leaving parochialism behind. South China Morning Post March 31.

\section{Nota}

1. Juego de palabras intraducible al español, entre la expresión G.I. Joe y el saludo Hi, Joe ("Hola, Joe»). N. del E. 